\title{
ĐÁNH GIÁ NĂNG LỰC CÁC PHÒNG THỬ NGHIỆM VI SINH THÔNG QUA CHƯƠNG TRİNH THỬ NGHIẸM THÀNH THẠO NĂM 2017
}

\author{
Nguyễn Thị Hằng ${ }^{1}$, Đoàn Thị Huờng \\ Viện Kiểm nghiệm an toàn vệ sinh thực phẩm Quốc gia
}

(Ngày đến tòa soạn: 28/6/2018; Ngày sủa bài sau phản biện: 8/8/2018; Ngày chấp nhận đăng: 17/8/2018)

\section{Tóm tắt}

TRONG xu thế họi nhập quốc tế WTO, ngày nay đảm bảo chất luợng trong phòng thư nghiệm 1 (PTN) là một yêu cầu quan trọng nhằm cung cấp các kết quả thử nghiệm đáng tin cây, có độ chính xác cao. Một trong các công cu đảm bảo chất luợng mà hiện nay được nhiều phòng thủ nghiệm áp dụng đó là việc tham gia thư nghiệm thành thạo (TNTT)/ so sánh liên phòng. Để đánh giá năng lực của các phòng thử nghiệm vi sinh trong cả nước, căn cú vào nhu cầu của các khách hàng, năm 2017 Viện Kiểm nghiệm an toàn vệ sinh thực phẩm Quốc gia đã tổ chức thành công 6 chương trình với các nền mẫu thực phẩm chung, thực phẩm chức năng. Thông qua việc xủ lý thống kê, tính hệ số z-score, Ban tổ chức đã đưa ra danh sách các phòng thử nghiệm vi sinh đạt yêu cầu khá cao nhu chi tiêu vi sinh vật hiếu khí, E.coli, Coliforms trên nền mẫu thực phẩm chức năng với tỉ lẹ đạt lần lượt là 92,9\%,100\%, 92,3\%. Tỷ lệ không đạt lớn nhất là chỉ tiêu nấm men nấm mốc trên nền mẫu thưc phẩm chức năng (35,7\%) và Coliforms trên mẫu nước (47,8\%). Thông qua kết quả này cho thấy năng lục các phòng thử nghiệm về vi sinh đã được tăng cả về chất lượng và số lương (chương trình có số đơn vị tham gia nhiều nhất là 89 phòng thư nghiệm).

Tù̀ khóa: Thử nghiệm thành thạo, so sánh liên phòng, z-score

\section{1. ĐẠT VẤN ĐỀ}

Hiện nay ở nước ta các phòng thử nghiệm được công nhận đạt tiêu chuẩn ISO 17025 ngày càng gia tăng, để thực hiện các yêu cầu của tiêu chuẩn này thì các cơ quan công nhận rất quan tâm đến kết quả tham gia thử nghiệm thành thạo của mỗi đơn vị khi đánh giá. Vì vậy, việc tổ chức các chương trình thử nghiệm thành thạo là cần thiết nhằm giúp các đơn vị có được bằng chứng để tự đánh giá năng lực kỹ thuật, đồng thời đây cũng là công cụ để các phòng thử nghiệm thực hiện chương trình đảm bảo chất lượng của mình.

Qua thăm dò thực tế về nhu cầu tham gia chương trình thử nghiệm thành thạo vi sinh của các đơn vị, năm 2017 Ban tổ chức thử nghiệm thành thạo của Viện Kiểm nghiệm an toàn vệ sinh thực phẩm Quốc gia (Viện) đã tổ chức được các chương trình như sau: Thử nghiệm thành thạo vi sinh trong thực phẩm chức năng (TPCN); Thử nghiệm thành thạo vi sinh trong nước; Thử nghiệm thành thạo vi sinh trong thực phẩm; Thử nghiệm thành thạo vi sinh trong sữa.

\section{CÁC BƯỚC CO’ BẢN TRONG TRIỂN KHAI CHƯƠNG TRÌNH THỦ NGHIỆM THÀNH THẠO}

\section{Lựa chọn và tạo mẫu}

Mẫu chuẩn bị từ nền mẫu tự nhiên sau đó cấy thêm chủng chuẩn bổ sung chất ổn định vi sinh rồi lắc trộn, đồng nhất kỹ ở điều kiện vô trùng. Mẫu đựng trong ống Fancol hoặc ampul, sau đó đặt trong túi thiếc hàn kín có dán nhãn và bảo quản ở nhiệt độ thích hợp.

\section{Gửi và phân tích mẫu}

Mẫu sau khi đóng gói và bảo quản đúng yêu cầu kỹ thuật sẽ được lấy ngẫu nhiên để kiểm tra

${ }^{1}$ Điện thoại: 0987041208 Email: nth202@gmail.com 
độ đồng nhất và độ ổn định.

Trong quá trình các đơn vị thực hiện phân tích mẫu, Ban tổ chức vẫn tiếp tục tiến hành đánh giá độ ổn định để đảm bảo các chỉ tiêu phân tích không bị biến đổi giữa các mẫu. Nếu kết quả độ ổn định đạt yêu cầu thì chương trình vẫn tiến hành bình thường. Nếu kết quả độ ổn định không đạt thì chương trình sẽ bị hủy.

\section{Báo cáo kết quả thử nghiệm}

Ban tổ chức thu thập kết quả của các phòng thử nghiệm và nhập dữ liệu vào máy tính. Để phân tích, xử lý số liệu, có thể sử dụng các phần mềm như: Excel, R.

Tính z-score theo 2 cách:

Cách 1: khoảng tú phân vị:

Tính $\mathrm{Q} 1, \mathrm{Q} 3$ và $\mathrm{IQR}=\mathrm{Q} 3-\mathrm{Q} 1, \mathrm{nIQR}=0,7413 \mathrm{nIQR}$

$$
\text { z-score }=\frac{\mathrm{LOG}_{10}(\text { Kết quả của PTN })-\text { Trung vị }}{n I Q R}
$$

Cách 2: tính theo giá trị ấn định $x *$ và độ lệch chuẩn $s^{*}$

$$
\text { z-score }=\frac{\text { Kết quả của PTN }-x^{*}}{s^{*}}
$$

Khi độ không đảm bảo đo của giá trị ấn định $\mathrm{u}\left(\mathrm{x}_{\mathrm{pt}}\right)>0,3 \sigma_{\mathrm{pt}}$ thì đánh giá kết quả theo hệ số $\mathrm{z}$-score

$$
z_{i}^{\prime}=\frac{x_{i}-x_{p t}}{\sqrt{\sigma_{p t}^{2}+u^{2}\left(x_{p t}\right)}}
$$

Căn cứ vào kết quả các phòng thử nghiệm, Ban tổ chức tiến hành xử lý số liệu và tính hệ số z-score hoặc z'-score, sau đó lập báo cáo trả các đơn vị:

\begin{tabular}{|c|l|}
\hline $\mid$ z-score $\mid$ hoặc $\mid$ z'-score $\mid \leq 2$ & Kết quả đạt yêu cầu \\
\hline $2<\mid$ z-score $\mid$ hoặc $\mid$ z'-score $\mid<3$ & Kết quả cảnh báo \\
\hline $\mid$ z-score $\mid$ hoặc $\mid$ z'-score $\mid \geq 3$ & Kết quả không đạt yêu cầu \\
\hline
\end{tabular}

\section{III. ĐÁNH GIÁ NĂNG LỬC CÁC PHÒNG THỬ NGHIẸM THAM GIA}

\section{Danh sách các chương trình TNTT vi sinh năm 2017 và số lượng PTN tham gia}

Trong năm 2017, Viện đã tổ chức được 6 chương trình thử nghiệm vi sinh trên nền mẫu thực phẩm, thực phẩm chức năng, nước và sữa như bảng 1 dưới đây.

Bảng 1. Danh sách các phòng thử nghiệm tham gia chuoong trình đánh giá

\begin{tabular}{|c|l|l|l|c|c|c|c|}
\hline TT & $\begin{array}{c}\text { Tên chương } \\
\text { trình }\end{array}$ & Chỉ tiêu & Mã số & $\begin{array}{c}\text { Số } \\
\text { PTN } \\
\text { tham } \\
\text { gia }\end{array}$ & $\begin{array}{c}\text { Số PTN } \\
\text { trong } \\
\text { hệ } \\
\text { thống } \\
\text { tế }\end{array}$ & $\begin{array}{c}\text { Số PTN } \\
\text { được } \\
\text { Bộ Y tế } \\
\text { chỉ } \\
\text { định } \\
(*)\end{array}$ & Ghi chú \\
\hline 1 & $\begin{array}{l}\text { TNTT vi sinh } \\
\text { trong TPCN }\end{array}$ & $\begin{array}{l}\text { Vi sinh vật hiếu khí } \\
\text { E.coli } \\
\text { Coliforms }\end{array}$ & V17.59 & 16 & 7 & 4 & $\begin{array}{c}\text { Chương } \\
\text { trình thu } \\
\text { phí }\end{array}$ \\
\hline
\end{tabular}




\begin{tabular}{|c|c|c|c|c|c|c|c|}
\hline TT & $\begin{array}{c}\text { Tên chương } \\
\text { trình }\end{array}$ & Chỉ tiêu & Mã số & $\begin{array}{l}\text { Số } \\
\text { PTN } \\
\text { tham } \\
\text { gia }\end{array}$ & $\begin{array}{c}\text { Số PTN } \\
\text { trong } \\
\text { hệ } \\
\text { thống y } \\
\text { tế }\end{array}$ & $\begin{array}{c}\text { Số PTN } \\
\text { được } \\
\text { Bộ Y tế } \\
\text { chî } \\
\text { định } \\
(*)\end{array}$ & Ghi chú \\
\hline 2 & $\begin{array}{l}\text { TNTT nấm men, } \\
\text { nấm mốc trong } \\
\text { TPCN }\end{array}$ & Nấm men, nấm mốc & V17.62 & 16 & 7 & 3 & $\begin{array}{l}\text { Chương } \\
\text { trình thu } \\
\text { phí }\end{array}$ \\
\hline 3 & $\begin{array}{l}\text { TNTT vi sinh } \\
\text { trong nước }\end{array}$ & $\begin{array}{l}\text { Coliforms } \\
\text { E.coli } \\
\text { Streptococcus } \\
\text { faecalis } \\
\text { Pseudomonas } \\
\text { aeruginosa }\end{array}$ & V17.76 & 24 & 18 & 5 & $\begin{array}{l}\text { Chương } \\
\text { trình thu } \\
\text { phí }\end{array}$ \\
\hline 4 & $\begin{array}{l}\text { TNTT vi sinh } \\
\text { trong thực phẩm }\end{array}$ & $\begin{array}{l}\text { Tổng số vsv hiếu khí } \\
\text { E.Coli } \\
\text { Staphylococci dương } \\
\text { tính với coagulase }\end{array}$ & V17.80 & 67 & 63 & 11 & $\begin{array}{l}\text { Chương } \\
\text { trình miễen } \\
\text { phí }\end{array}$ \\
\hline 5 & $\begin{array}{l}\text { TNTT vi sinh } \\
\text { trong sữa }\end{array}$ & $\begin{array}{l}\text { Listeria } \\
\text { monocytogens } \\
\text { Enterobacteriace }\end{array}$ & V17.85 & 30 & 14 & 10 & $\begin{array}{l}\text { Chương } \\
\text { trình miễn } \\
\text { phí }\end{array}$ \\
\hline 6 & $\begin{array}{l}\text { TNTT vi sinh } \\
\text { trong thực phẩm }\end{array}$ & $\begin{array}{l}\text { Tổng số vsv hiếu khí } \\
\text { Tổng NM-NM } \\
\text { B.cereus }\end{array}$ & V17.86 & 89 & 51 & 15 & $\begin{array}{l}\text { Chương } \\
\text { trình miễn } \\
\text { phí }\end{array}$ \\
\hline
\end{tabular}

Ghi chú: (*) Đơn vị được Bộ Y tế chỉ định theo TT 20/2013/TTLT-BYT-BCT-BNNPTNN

Đồ thị biểu diễn số lượng các PTN tham gia thể hiện trong hình 1:

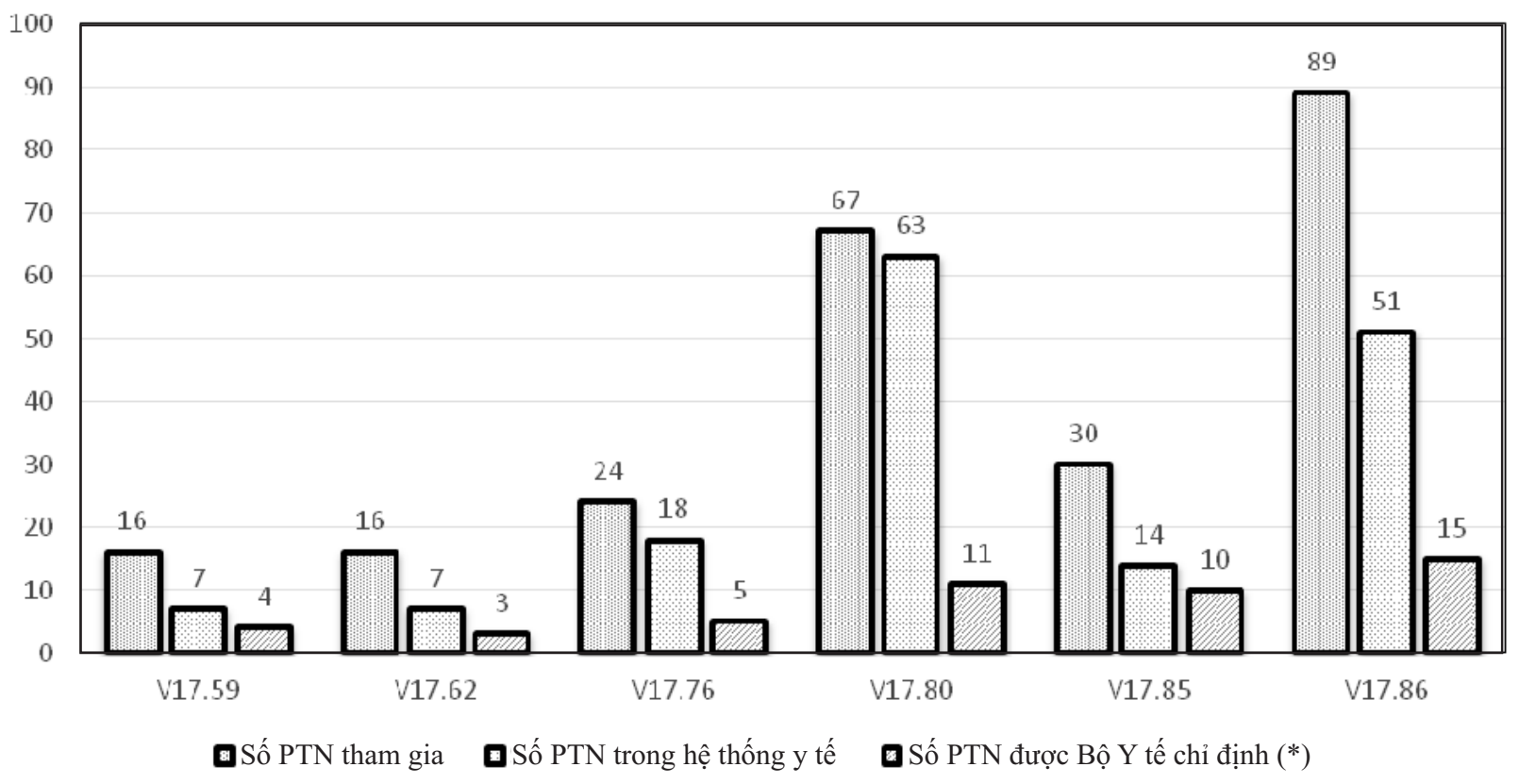

Hình 1. Đồ thị biểu diễn các PTN tham gia đối với mỗi chuơng trình

Từ đồ thị Hình 1 cho thấy các đơn vị tham gia nhiều nhất là chương trình vi sinh trong thực phẩm với số lượng 89 phòng. Đây là chương trình có nền mẫu thực phẩm thông thường, với các chỉ 
tiêu vi sinh cơ bản do vậy thích hợp với đa số các phòng thử nghiệm, đặc biệt các đơn vị thuộc hệ thống quản lý của Bộ Y tế.

Một số chương trình có số phòng tham gia hạn chế là chương trình vi sinh trong thực phẩm chức năng và nước, đây là chương trình thu phí đồng thời có chỉ tiêu phân tích khó như Streptococcus faecalis, Pseudomonas aeruginosa nên thu hút ít các đơn vị tham gia.

\section{2. Đánh giá năng lực các phòng thử nghiệm vi sinh}

Ban tổ chức căn cứ tiêu chuẩn ISO 13528:2015 và ISO 17043:2010 để tính hệ số Z-score đối với các chỉ tiêu. Tỷ lệ các phòng thử nghiệm đạt hay không đạt yêu cầu hoặc cảnh báo được thể hiện trong bảng 2 .

Bảng 2. Số liệu thống kê đánh giá năng lực các phòng thư nghiệm

\begin{tabular}{|c|c|c|c|c|c|c|c|}
\hline \multirow[b]{2}{*}{$S T T$} & \multirow[b]{2}{*}{ Mã số } & \multirow[b]{2}{*}{ Tên chì tiêu } & \multicolumn{5}{|c|}{ Số PTN } \\
\hline & & & Tổng & $\begin{array}{l}\text { Báo } \\
\text { hüy }\end{array}$ & Dat $(\%)$ & $\begin{array}{c}\text { Cảnh báo } \\
\text { (\%) }\end{array}$ & $\begin{array}{l}\text { Không } \\
\text { dat (\%) }\end{array}$ \\
\hline \multirow{3}{*}{1} & \multirow{3}{*}{ V17.59-1 } & Tổng số vsv hiếu khí & 16 & 2 & 85,7 & 7,1 & 7,1 \\
\hline & & E.coli & 14 & 2 & 91,7 & 0 & 8,3 \\
\hline & & Coliforms & 15 & 2 & 76,9 & 7,7 & 15,4 \\
\hline \multirow{3}{*}{2} & \multirow{3}{*}{ V17.59-2 } & Tổng số vsv hiếu khí & 16 & 2 & 92,9 & 7,1 & 0 \\
\hline & & E.coli & 14 & 2 & 100 & 0 & 0 \\
\hline & & Coliforms & 15 & 2 & 92,3 & 7,7 & 0 \\
\hline 3 & V17.62-1 & $\begin{array}{l}\text { Tổng số nấm men, nấm } \\
\text { mốc }\end{array}$ & 14 & 0 & 64,3 & 0 & 35,7 \\
\hline 4 & V17.62-2 & $\begin{array}{l}\text { Tổng số nấm men, nấm } \\
\text { mốc }\end{array}$ & 15 & 0 & 73,3 & 13,3 & 13,3 \\
\hline \multirow{4}{*}{5} & \multirow{4}{*}{ V17.76-1 } & Coliforms & 23 & 1 & 81,8 & 0 & 18,2 \\
\hline & & E.coli & 23 & 1 & 77,3 & 0 & 22,7 \\
\hline & & Streptococcus faecalis & 15 & 1 & 92,9 & 0 & 7,1 \\
\hline & & Pseudomonas aeruginosa & 18 & 1 & 94,1 & 0 & 5,9 \\
\hline \multirow{4}{*}{6} & \multirow{4}{*}{ V17.76-2 } & Coliforms & 24 & 1 & 52,2 & 0 & 47,8 \\
\hline & & E.coli & 24 & 1 & 100 & 0 & 0 \\
\hline & & Streptococcus faecalis & 16 & 1 & 93,3 & 0 & 6,7 \\
\hline & & Pseudomonas aeruginosa & 19 & 1 & 94,4 & 0 & 5,6 \\
\hline \multirow{3}{*}{7} & \multirow{3}{*}{ V17.80-1 } & E. Coli & 61 & 1 & 68,3 & 6,7 & 25 \\
\hline & & Tổng số vsv hiếu khí & 64 & 1 & 61,9 & 9,5 & 28,6 \\
\hline & & $\begin{array}{l}\text { Staphylococci dương tính } \\
\text { với coagulase }\end{array}$ & 59 & 1 & 86,2 & 8,6 & 5,2 \\
\hline \multirow{3}{*}{8} & \multirow{3}{*}{ V17.80-2 } & E. Coli & 60 & 1 & 67,8 & 13,6 & 18,6 \\
\hline & & Tổng số vsv hiếu khí & 64 & 1 & 58,7 & 6,3 & 34,9 \\
\hline & & $\begin{array}{l}\text { Staphylococci dương tính } \\
\text { với coagulase }\end{array}$ & 59 & 1 & 79,3 & 0 & 20,7 \\
\hline \multirow{2}{*}{9} & \multirow{2}{*}{ V17.85-1 } & Listeria monocytogens & 27 & 4 & 78,3 & 0 & 21,7 \\
\hline & & Enterobacteriace & 29 & 4 & 84 & 0 & 16 \\
\hline \multirow{2}{*}{10} & \multirow{2}{*}{ V17.85-2 } & Listeria monocytogens & 26 & 4 & 81,8 & 0 & 18,2 \\
\hline & & Enterobacteriace & 29 & 4 & 76 & 12 & 12 \\
\hline \multirow{3}{*}{11} & \multirow{3}{*}{ V17.86-1 } & Tổng số vsv hiếu khí & 88 & 3 & 72,9 & 3,5 & 23,5 \\
\hline & & $\begin{array}{l}\text { Tổng số nấm men, nấm } \\
\text { mốc }\end{array}$ & 83 & 3 & 72,5 & 12,5 & 15 \\
\hline & & B.cereus & 66 & 3 & 96,8 & 3,2 & 0 \\
\hline \multirow{3}{*}{12} & \multirow{3}{*}{ V17.86-2 } & Tổng số vsv hiếu khí & 89 & 3 & 90,7 & 5,8 & 3,5 \\
\hline & & $\begin{array}{l}\text { Tổng số nấm men, nấm } \\
\text { mốc }\end{array}$ & 88 & 3 & 84,7 & 2,4 & 12,9 \\
\hline & & B.cereus & 65 & 3 & 90,3 & 0 & 9,7 \\
\hline
\end{tabular}


Kết quả tham gia TNTT về lĩnh vực vi sinh trên nền mẫu thực phẩm, thực phẩm chức năng, sữa và nước năm 2017 thể hiện ở các Hình $2,3,4$ và 5 .

• Đối với nền mẫu thực phẩm thông thường

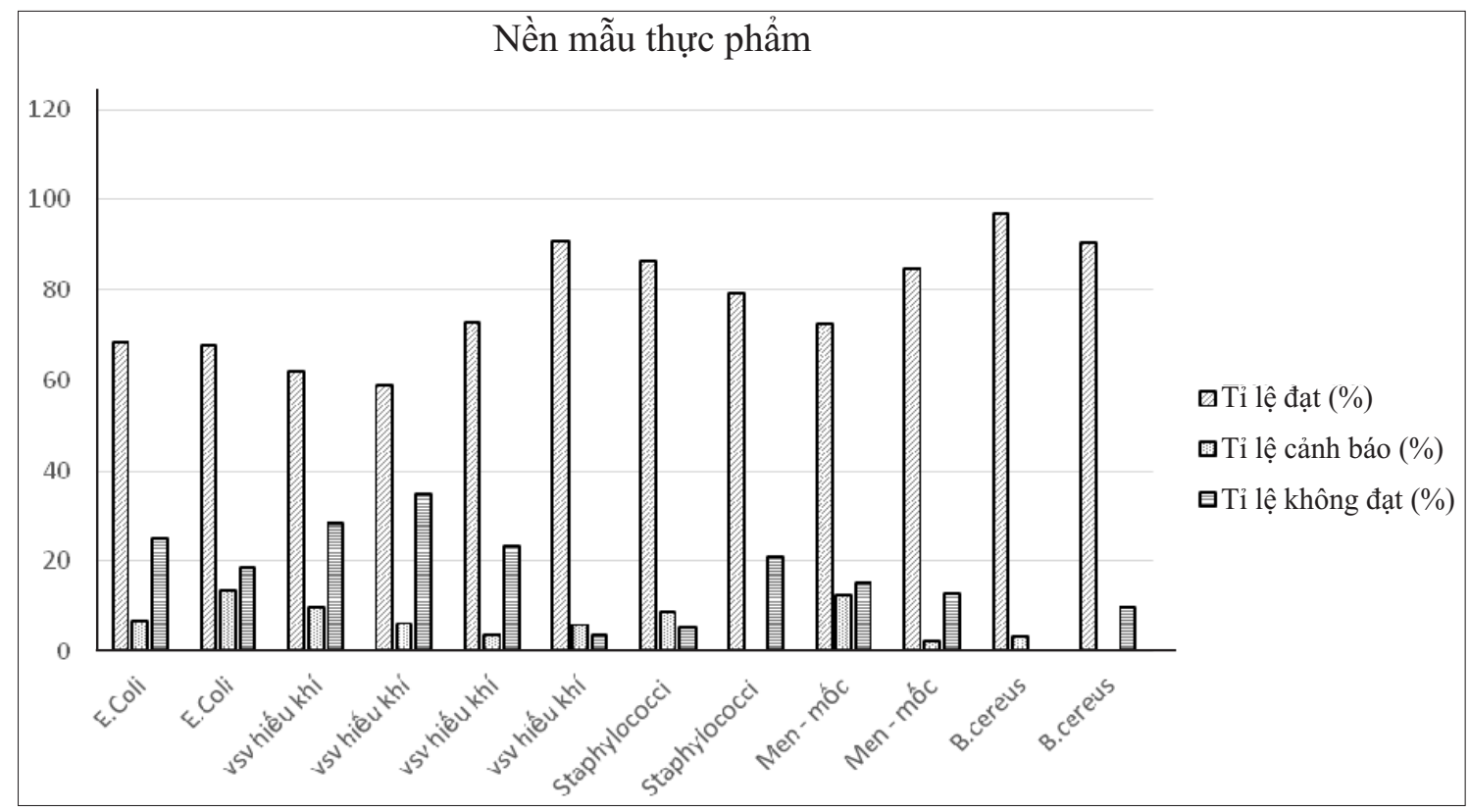

Hình 2. Đồ thị biểu diễn kết quả của các PTN (\%) đối với nền mẫu thực phẩm

Kết quả ở Hình 2 cho thấy tỷ lệ các PTN đạt yêu cầu rất cao như chỉ tiêu B.cereus là $96,8 \%$ và $90,3 \%$. Tuy nhiên, chỉ tiêu tổng vi sinh vật hiếu khí là chỉ tiêu đơn giản nhưng tỷ lệ cảnh báo là 23,5 $\%$ và $34,9 \%$ là khá cao. Chỉ tiêu $E$. coli không đạt yêu cầu chiếm tỷ lệ $6,7 \%-13,6 \%$ và cảnh báo là $18,6 \%-25 \%$, tỷ lệ đạt khá khiêm tốn chỉ ở mức hơn $60 \%$.

- Đối với nền mẫu thực phẩm chức năng

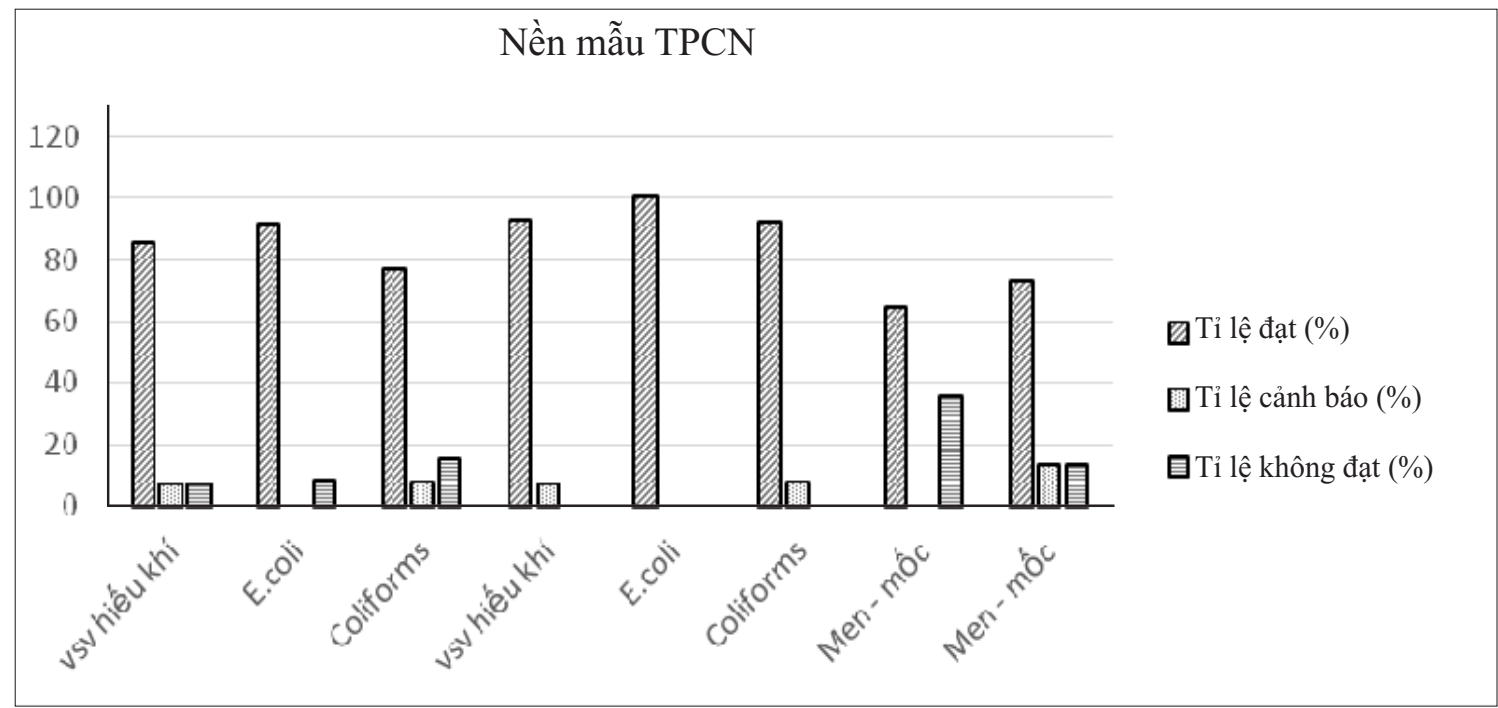

Hình 3. Đồ thị biểu diễn kết quả của các PTN tham gia (\%) đối với nền mẫu TPCN

Đối với nền mẫu thực phẩm chức năng (TPCN), chỉ tiêu đạt cao nhất là E.coli sau đó là vi khuẩn hiếu khí và Coliforms. Chỉ tiêu nấm men - nấm mốc có tỷ lệ cảnh báo là $13,3 \%$ đến $35,7 \%$, con số này đòi hỏi các phòng thử nghiệm cần xem xét lại điều kiện phân tích, phương pháp phân tích hoặc môi trường, hóa chất, tay nghề phân tích,... vì đây là chỉ tiêu vi sinh cơ bản. 
• Đối với nền mẫu nước

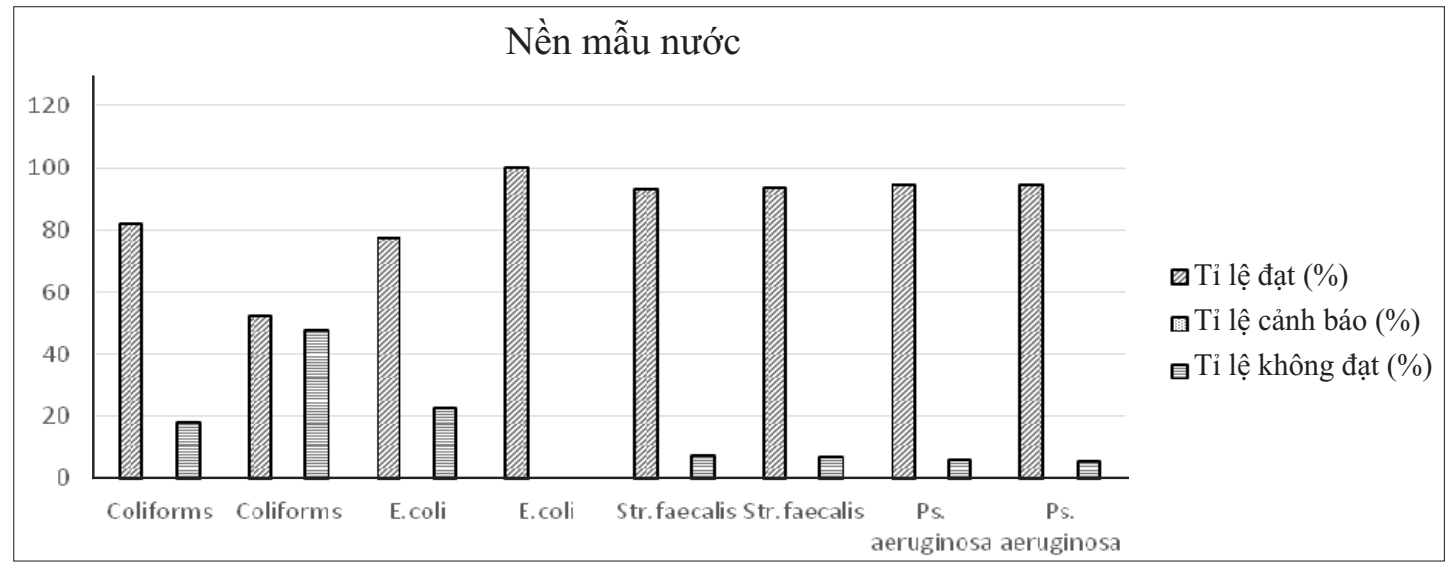

Hình 4. Đồ thị biểu diễn kết quả của các PTN tham gia (\%) đối với nền mẫu nuớc

Đối với nền mẫu nước chỉ tiêu đạt yêu cầu cao nhất là $E$.coli từ $86,6 \%$ đến $100 \%$. Tuy nhiên chỉ tiêu Colifoms chỉ đạt yêu cầu ở mức $52,2 \%$ và cảnh báo ở $47,8 \%$, điều này cho thấy các phòng thử nghiệm cần phải xem xét lại vì đây là chỉ tiêu thông dụng và phổ biến trong các phòng thử nghiệm vi sinh.

• Đối với nền mẫu sũa

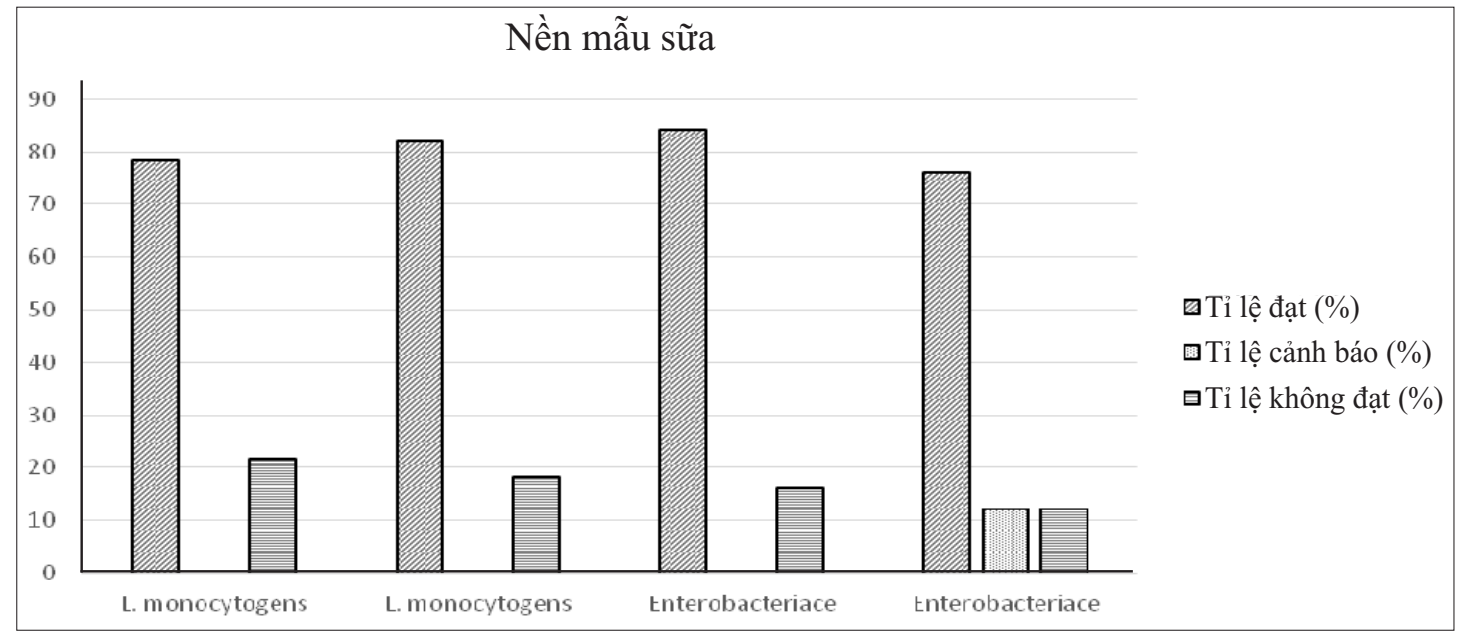

Hình 5. Đồ thị biểu diễn kết quả của các PTN tham gia (\%) đối với nền mẫu sũa

Chương trình này có tỷ lệ đạt khá cao với chỉ tiêu L.monocytogens đạt từ $78,3-81,8 \%$, chỉ tiêu Enterobacteriace đạt từ 76- 84\%. Tuy nhiên một số phòng thử nghiệm ở mức cảnh báo, có thể do phương pháp hoặc tay nghề nhân viên chưa cao nên có sự nhầm lẫn trong việc nhận biết các vi khuẩn.

\section{NHẬN XÉT VÀ BÀN LUẬN}

Trong năm 2017, Viện đã tổ chức 6 chương trình TNTT về lĩnh vực vi sinh trên các nền mẫu thực phẩm, nước, thực phẩm chức năng và sữa với các chỉ tiêu vi sinh cơ bản (tổng số vi sinh vật hiếu khí, nấm men - nấm mốc, B.cereus,..), vi sinh gây bệnh (L.monocytogens) và các chỉ tiêu chỉ điểm vệ sinh (E.coli, Coliforms,..). Trong đó, các PTN tham gia trên nền mẫu thực phẩm nhiều hơn đối với nền mẫu thực phẩm chức năng và nước. Tỉ lệ tham gia của các phòng được chỉ định theo Thông tư số 20/2013/TTLT-BYT-BCT-BNNPTNN còn hạn chế, có thể do một số chương trình thu phí.

Một số phòng thử nghiệm chỉ đăng ký tham gia chương trình miễn phí nên cần có sự đầu tư nguồn lực để tham gia nhiều hơn và từ đó mới có thể đánh giá năng lực của đơn vị mình một cách chính xác nhằm có những cải tiến kịp thời. 
Một số chương trình có chỉ tiêu thông thường nhưng tỷ lệ đạt không cao và tỷ lệ cảnh báo nhiều nên các phòng cần tìm nguyên nhân để khắc phục.

Đối với chương trình vi sinh, Ban tổ chức thường làm mẫu cặp đôi, cùng một chỉ tiêu nhưng ở 2 mẫu khác nhau, cùng một phòng thử nghiệm thực hiện thì có mẫu đạt, mẫu không đạt hoặc cảnh báo, điều này chứng tỏ tay nghề của các kiểm nghiệm viên chưa thuần thục.

Một số phòng thử nghiệm có chỉ tiêu không đạt hoặc ở mức cảnh báo cần xem xét một vài nguyên nhân như sau:

- Thiết bị: các thiết bị phân tích phải được hiệu chuẩn định kỳ, chỉ thực hiện phép thử trên các thiết bị đã được hiệu chuẩn và bảo dưỡng đúng theo quy định.

- Các môi trường, hóa chất, chủng chuẩn phải có xuất xứ nguồn gốc và còn hạn sử dụng.

- Quy trình phân tích phải được thẩm định và là quy trình chuẩn.

- Điều kiện tiện nghi môi trường đảm bảo đáp ứng theo yêu cầu của phòng thử nghiệm vi sinh.

- Báo cáo kết quả tuân theo yêu cầu của Ban tổ chức về đơn vị và cách biểu thị kết quả.

- Thông thường với mỗi chương trình thử nghiệm thành thạo vi sinh, Ban tổ chức sẽ gửi 2 mẫu đồng thời nên các phòng thử nghiệm cần chú ý trong cả quá trình bảo quản, xử lý và phân tích mẫu tránh việc nhầm lẫn giữa 2 mẫu, đặc biệt đối với các chương trình định tính.

\section{KẾT LUÂN}

Việc tham gia thử nghiệm thành thạo để khẳng định kết quả đối với các phòng thử nghiệm là rất cần thiết. Các chương trình thử nghiệm thành thạo của Viện tổ chức đã tăng cả về chất lượng và số lượng, cũng như đa dạng các nền mẫu. Các phòng thử nghiệm tham gia đạt tỉ lệ đạt cao với các chỉ tiêu vi sinh cơ bản (vi sinh vật hiếu khí, B.cereus,..) trên nền mẫu thực phẩm và các chỉ tiêu chỉ điểm vệ sinh trên nền mẫu thực phẩm chức năng. Tuy nhiên, trên nền mẫu nước, tỉ lệ các phòng thử nghiệm không đạt lớn như Coliforms là 47,8\%.

\section{TÀI LIÊU THAM KHẢO}

1. ISO 17043:2010, Conformity assessment. General requirements for proficiency testing.

2. ISO 13528:2015 Statistical methods for use in proficiency testing by interlaboratory comparison

3. ISO/IEC 17025:2017, General requirements for the competence of testing and calibration laboratories.

4. APL 03, Văn phòng công nhận chất lượng Việt Nam, (2016), Chính sách về Thử nghiệm thành thạo/So sánh liên phòng.

5. R103, American Association for Laboratory Accreditation, (2013), General requirements: Proficiency testing for ISO/IEC 17025 laboratories.

\section{Summary}

\section{EVALUATION THE CAPACITY OF MICROBIOLOGICAL LABORATORIES THROUGH PROFICIENCY TESTING PROGRAMS IN 2017}

\section{Nguyen Thi Hang, Doan Thi Huong}

National Institute for Food Control

In the context of international integration into the WTO, quality assurance in laboratories is an important requirement for providing reliable and highly accurate test results. One of the quality assurance tools currently used by various laboratories is the participation in proficiency testing (TNTT)/ interlaboratory comparisons. In order to assess the capacity of microbiological laboratories throughout the country and respond to the requirements of the customers, the National Institute for Food Control (NIFC) organized 6 biological proficiency testing schemes in food and functional foods in 2017. Based on the statistical processing and z-score calculation, NIFC has made a list of highly satisfactory microbiological laboratories such as such the pass rate of Total Plate Count, E. coli, Coliforms in functional food with $92.9 \%, 100 \%, 92.3 \%$... The highest failure rate of Yeast and Mould in functional food (35.7\%) and Coliforms in water (47.8\%)... The results showed that the capacity of microbiology laboratories has been increased in both quality and quantity (with the largest number of participants of 89 laboratories).

Keywords: Proficiency testing, interlaboratory comparisons, z-score 